\title{
Postoperative Delirium After Liver Transplantation is Associated With Increased Length of Stay and Lower Survival in a Prospective Cohort
}

Progress in Transplantation 2017, Vol. 27(I) 23-30

(C) 2016, NATCO. All rights reserved. Reprints and permission: sagepub.com/journalsPermissions.nav DOI: I0.II77/I5269248|6679838 journals.sagepub.com/home/pit (S)AGE

\author{
Sonja Beckmann, MScN, RN ${ }^{1,2}$, Maria Schubert, PhD, $R N^{3,4}$, \\ Hanna Burkhalter, PhD, RN ${ }^{5}$, Philipp Dutkowski, MD ${ }^{6}$, and \\ Sabina De Geest, PhD, RN ${ }^{1,7}$
}

\begin{abstract}
Introduction: Although postoperative delirium (POD) is a common in-hospital complication associated with negative outcomes, evidence in liver transplantation (LT) is scarce. Objective: We examined the incidence and duration of POD, its impact on outcomes and health-care utilization, and described predisposing and precipitating factors favoring POD development. Design: This prospective cohort study included adults undergoing transplantation in a tertiary hospital. Postoperative delirium was assessed 3 times daily until 24 days post-LT, with the Intensive Care Delirium Screening Checklist on the intensive care unit (ICU) and the Delirium Observation Screening Scale on the ward. Postoperative delirium was noted if any of the daily measurements was positive. Results: Forty-two patients $(69 \%$ male, mean age 55 years, median Model for End-Stage Liver Disease score 15 [interquartile range 8-26]) were included. The incidence of POD was $45.2 \%$, with a median duration of 5 days. Patients with POD had longer ICU (median 8 vs 2 days, $P=.000$ ) and hospital stays (median 32 vs 14 days, $P=.000$ ) as well as shorter survival (Breslow test $P=.045$, log-rank test $P=.150$ ). Pre-LT comorbidities and perioperative factors might be related to POD development. Conclusion: Nurses are key persons in the detection of POD in the daily clinical routine. The high incidence of POD and its negative association with patient outcomes highlight not only the relevance of systematic assessment of POD after LT but also the need for preventive interventions.
\end{abstract}

\section{Keywords}

health-care utilization, postoperative complication, mortality, intensive care unit, nursing care

\section{Introduction}

Postoperative delirium (POD), a common in-hospital complication, is associated with negative outcomes, for example, mortality, increased length of stay, and functional decline. ${ }^{1}$ Severely ill patients are at higher risk of developing POD and the incidence ranges from $19 \%$ to $82 \% .{ }^{1}$ Considering that organ allocation follows the Model for End-Stage Liver Disease (MELD), patients receiving liver transplantation (LT) are typically severely ill at the time of transplantation. Since the implementation of MELD, patients have had increased morbidity following LT. ${ }^{2}$ Therefore, prevention of additional complication such as POD is crucial. While predisposing and precipitating risk factors contribute independently to POD's development, their interaction can also aggravate its progress. ${ }^{3}$ Indicating a patient's baseline vulnerability, predisposing factors include, for example, older age, alcohol misuse, and comorbidities such as depression. ${ }^{1}$ Precipitating factors occur after hospital admission and include, for example, surgery, polypharmacy, and physical restraints. ${ }^{1}$
Delirium is defined as a change in cognition, along with disturbances of attention and awareness, characterized by reduced capacity to direct, focus, sustain, or shift attention as

\footnotetext{
I Institute of Nursing Science, University of Basel, Basel, Switzerland

${ }^{2}$ Department of Abdomen-Metabolism, University Hospital Zurich, Zurich, Switzerland

${ }^{3}$ Center of Clinical Nursing Science, University Hospital Zurich, Zurich, Switzerland

${ }^{4}$ Directorate of Nursing/MTT, Insel Group AG, University Hospital Insel Bern, Bern, Switzerland

${ }^{5}$ Center for Sleep Medicine, Hirslanden Group, Zollikon, Switzerland

${ }^{6}$ Department of Surgery and Transplantation, University Hospital Zurich, Zurich, Switzerland

${ }^{7}$ Academic Center for Nursing and Midwifery, Department of Public Health and Primary Care, KU Leuven, Belgium
}

Corresponding Author:

Sabina De Geest, Institute of Nursing Science, University of Basel, Bernoullistrasse 28, 4056 Basel, Switzerland.

Email: sabina.degeest@unibas.ch 
Progress in Transplantation 27(I)

well as reduced orientation to the environment. ${ }^{4}$ However, its tendencies to develop quickly and fluctuate in severity over the course of a day handicap observation-based recognition. ${ }^{4}$ Nurses are major providers of bedside care and take a leading role in the detection of delirium. They may use a broad variety of valid and reliable instruments to assess delirium, ${ }^{5}$ which also precludes the risk of underrecognition of delirium as clinical diagnosis. ${ }^{1,6}$ About $30 \%$ to $40 \%$ of delirium episodes are preventable with (non) pharmacological interventions, which highlights the importance of monitoring the development of POD by using assessment tools. ${ }^{1}$

The few researchers who examined POD in the LT population have reported incidences ranging from $9.8 \%$ to $47.4 \%$. $^{7-13}$ However, the majority assessed delirium only as one of a cluster of neurological complications. Examining POD as a primary post-LT outcome, though, ${ }^{7,12} 2$ recent studies associated it with increased in-hospital and 1-year mortality, increased length of intensive care unit (ICU) and hospital stays, longer duration of mechanical ventilation, and higher rates of infection or sepsis. ${ }^{7,12}$ However, both studies used retrospective designs and focused on the occurrence of POD in the ICU without further assessment on the ward. Follow-up time was unclear ${ }^{12}$ or limited to 1 -year posttransplantation. ${ }^{7}$ Wang et al used a validated instrument to assess POD and identified a history of alcohol abuse, preoperative hepatic encephalopathy, prolonged endotracheal intubation, and an Acute Physiology and Chronic Health Evaluation II score $\geq 16$ as risk factors for the development of POD. ${ }^{12}$ As they included only living-donor LT recipients, its evidence is not generalizable across the entire LT population. To date, no prospective studies have used validated assessment instruments to measure POD in a representative group of LT recipients. Therefore, the aims of this study were (1) to examine the incidence and duration of POD in LT recipients during their hospital stay, (2) to examine the impact of POD on clinical outcomes (ie, survival and postoperative complications) and healthcare utilization (ie, ICU and hospital length of stay), and (3) to describe potential predisposing and precipitating factors potentially favoring the development of POD.

\section{Methods}

\section{Design, Setting, and Population}

This prospective cohort study was conducted in the ICU for visceral and transplant surgery and the transplant ward of the University Hospital Zurich, where about 50 LTs per year are conducted. ${ }^{14}$ All German-speaking adults ( $>18$ years) awaiting single-organ LT and competent to provide informed consent were eligible. The primary investigator contacted all patients on the waiting-list. Those eligible and interested received oral and written study information. Written informed consent prior to transplantation was mandatory before inclusion. Recruitment began in December 2011; data collection lasted from February 2012 (first participant admitted for LT) until June 2014 (end of study period). In-hospital follow-up lasted until maximum 24 days post-LT, earlier discharge, or death. The primary investigator collected data from patients' medical files and randomly error checked $10 \%$ of the entered data with a research assistant. Data were kept confidential, with access restricted to members of the research team. The study was approved by the Cantonal Ethics Committee of Zurich (KEKZH-Nr. 2011-0317).

\section{Data Collection}

Demographic and clinical data including recipient and donor ages, gender, nationality, marital status, mode of hospital admission, etiology of end-stage liver disease, first or re-LT, type of donation, and cold ischemia time were collected from the patients' medical files.

\section{Measurement of POD}

In the ICU, POD was assessed with the German version of the 8 -item Intensive Care Delirium Screening Checklist. ${ }^{15,16}$ This tool is validated to screen delirium in critically ill patients by systematic observation. Each item is rated as no ( 0 points), yes (1 point), or not assessable (no scoring) and summed to a maximum score of 8 , with scores $\geq 4$ indicating delirium. As the Intensive Care Delirium Screening Checklist is only applicable to patients who are awake and respond to stimulation, consciousness in critically ill patients must first be screened using the Richmond Agitation Sedation Scale. ${ }^{17}$ Each patient's level of agitation is scored on a 10-point scale (range: -5 to +4 ). As patients with scores of -5 or -4 are deeply sedated or unarousable, only patients scoring between -3 and +4 are eligible for POD assessment.

During the postoperative follow-up period, patients on the ward were screened using the 13-item Delirium Observation Screening Scale, which enables nurses to detect early signs of delirium observable during regular care. ${ }^{18,19}$ All items are rated as sometimes/always ( 1 point), never ( 0 point), or don't know (no scoring) and summed for a final score. A score $\geq 3$ indicates delirium.

The systematic delirium assessment was incorporated into the clinical routines in January 2012 in the context of the hospital's Delir-Path project, a multiprofessional practice development and health service research project. The multicomponent delirium management protocol includes delirium screening as well as measures to prevent and treat delirium in acute care patients. All nurses and physicians received training to apply the assessment instruments (M. Schubert $\mathrm{PhD}$ and $\mathrm{R}$. Spirig PhD Delir-Path: Detect Evaluate Control Inpatient Risk factors, Prevent and Treat Hospital Acquired Deliriums (Health Services Research Project). University Hospital Zurich. Unpublished; 2013:1-22.). In both settings, starting on the first full day post-LT and continuing to a maximum of 24 days post-LT, bedside nurses screened patients in each of the 3 shifts/d for POD, documenting the results in the medical files. As differentiation between POD and effects of anesthesia can be problematic, patients were not assessed for POD on the day 
Table I. Overview of Predisposing and Precipitating Factors Potentially Favoring the Development of POD After LT.

\begin{tabular}{|c|c|c|c|}
\hline \multicolumn{2}{|l|}{ Variables } & \multirow{2}{*}{$\begin{array}{l}\text { Definition } \\
\text { In years at the time of } L T\end{array}$} & \multirow{2}{*}{$\begin{array}{l}\text { Time of Data Collection } \\
\text { Once }\end{array}$} \\
\hline Predisposing factors & Recipient age & & \\
\hline \multirow[t]{3}{*}{ before LT } & MELD & $\begin{array}{l}\text { Calculated MELD score (range 6-40) at the time of LT as } \\
\text { mentioned in the medical files }\end{array}$ & Once \\
\hline & Comorbidities & $\begin{array}{l}\text { Pre-LT comorbidities (presence or absence) as mentioned in the } \\
\text { medical files: Diabetes mellitus, history of depression, or need } \\
\text { of renal replacement therapy (hemodialysis or hemofiltration) }\end{array}$ & Once \\
\hline & Laboratory variables & $\begin{array}{l}\text { Test results from the most recent blood withdrawal before LT: } \\
\text { total bilirubin, sodium, alanine aminotransferase, aspartate } \\
\text { aminotransferase }\end{array}$ & Once \\
\hline \multirow{5}{*}{$\begin{array}{l}\text { Precipitating factors at } \\
\text { the time of LT or } \\
\text { afterward }\end{array}$} & $\begin{array}{l}\text { Intraoperative } \\
\text { hypotension }\end{array}$ & $\begin{array}{l}\text { Systolic blood pressure }<90 \mathrm{mmHg} \text { at any time during surgery } \\
\text { (presence or absence) as mentioned in the medical files }\end{array}$ & Once \\
\hline & Duration of surgery & $\begin{array}{l}\text { Duration of the LT surgery (in minutes) as mentioned in the } \\
\text { medical files }\end{array}$ & Once \\
\hline & $\begin{array}{l}\text { Renal replacement } \\
\text { therapy }\end{array}$ & $\begin{array}{l}\text { Hemodialysis or hemofiltration after LT (presence or absence) as } \\
\text { mentioned in the medical files }\end{array}$ & Once \\
\hline & Blood transfusion & $\begin{array}{l}\text { Median amount of red blood cell transfusions (in liter) from } \\
\text { beginning of surgery until the end of in-hospital follow-up }\end{array}$ & $\begin{array}{l}\text { Daily, until maximum of } \\
\text { 24th day after } L T\end{array}$ \\
\hline & Medication & $\begin{array}{l}\text { Mean amount (in mcg or mg) of the applied medication per } \\
\text { patient after LT: opioid (fentanyl, morphine); sedative } \\
\text { (propofol, midazolam); immunosuppressive agent } \\
\text { (solumedrol, }{ }^{\mathrm{a}} \text { prednisone, tacrolimus, cyclosporine, } \\
\text { mycophenolate mofetil) }\end{array}$ & $\begin{array}{l}\text { Daily, until maximum of } \\
\text { 24th day after LT }\end{array}$ \\
\hline
\end{tabular}

Abbreviations: MELD, Model for End-Stage Liver Disease; LT, liver transplantation; ICU, intensive care unit; POD, postoperative delirium.

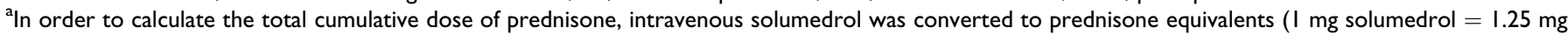
prednisone).

of surgery. For our study, the presence of POD on that day was defined if any of the 3 daily measurements indicated delirium.

\section{Patient Outcomes and Health-Care Utilization}

Patient survival time was defined as time from LT to death or the end of study period (June 2014). Length of ICU stay was measured starting the first full day after surgery until discharge to the ward or death (if it occurred in the ICU). Length of hospital stay was measured from the first full day posttransplant until discharge from hospital or death (if death occurred before discharge). Postoperative complications were defined according to the Classification of Surgical Complications ${ }^{20}$ and pooled into 4 categories: mild (grades I and II), moderate (grade III), severe (grade IV), and death (grade V). If more than one complication occurred, the highest category mentioned in the medical files was noted.

\section{Predisposing and Precipitating Factors}

Factors were categorized according to Inouye and Charpentier's model of delirium etiology. ${ }^{3}$ The selection of specific factors was based on the literature, practicability, and clinical relevance to LT recipients (Table 1). Data were prospectively collected from the medical files.

\section{Data Analysis}

Descriptive statistics (frequency, percentage, mean, standard deviation [SD], median, and interquartile ranges [IQR]) were calculated as appropriate based on measurement levels and data distributions. The cohort was divided into 2 groups defined by the presence or absence of POD at any time. To examine POD's impact on clinical outcomes and to compare potential predisposing and precipitating factors, intergroup comparisons were performed via Fisher exact test for categorical variables and via the Mann-Whitney $U$ test for continuous variables. To explore the impact of POD on patient survival, Kaplan-Meier analysis was performed for death, censored after follow-up completion. Differences in survival between patients with and without POD were examined with the Log-Rank test and the Wilcoxon-Breslow test, which is more sensitive to earlier events. ${ }^{21}$ A 2 -tailed $P$ value $<.05$ was considered statistically significant. Statistical analysis was performed using SPSS for Mac, release version 21 (IBM SPSS Inc, Armonk, New York).

\section{Results}

\section{Sample Characteristics}

During the study period, 197 patients were registered on the LT waiting-list, whereof 122 could be checked up on the inclusion 


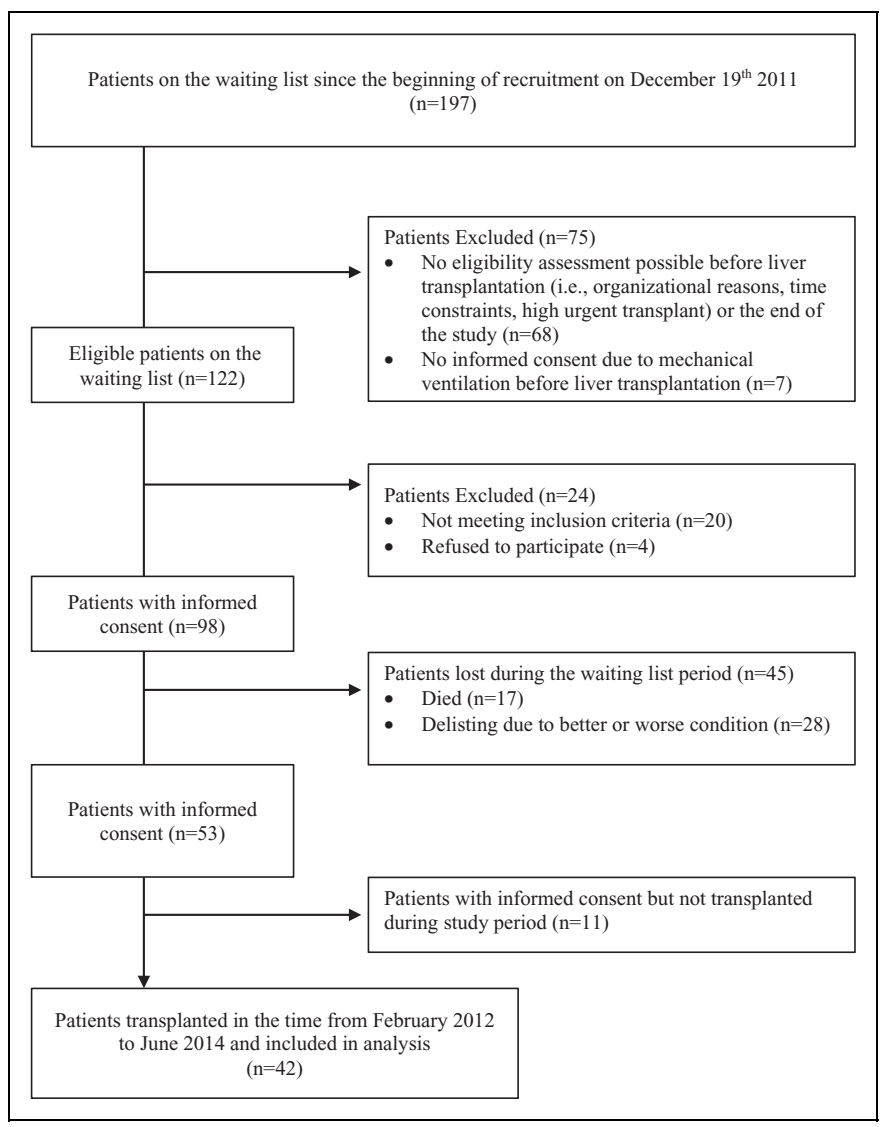

Figure I. Flowchart of the recruitment, inclusion, and exclusion of patients during the study period.

criteria. Of the 98 patients who were enrolled in this study, 45 were excluded from the waiting-list due to progression of the underlying disease or death before LT, 11 were still on the waiting-list at the end of the study, while 42 finally received transplants (flowchart Figure 1). The cohort was followed for a median duration of 531 days (range 30-840 days). The sample was predominantly male $(\mathrm{n}=29,69 \%)$ and Swiss $(\mathrm{n}=30$, $71.4 \%)$, with a mean age of $55(\mathrm{SD}=10.9$ years). At $\mathrm{LT}$, the median calculated MELD score was 15 (IQR 8-26), and the most common end-stage liver disease was hepatocellular carcinoma $(\mathrm{n}=17,40.5 \%)$. Most patients were admitted to hospital from home $(n=29,69 \%)$. Four $(9.5 \%)$ organs were derived from living donors. Our analyses revealed no relationships between sample characteristics and the presence or absence of POD (Table 2).

\section{Incidence and Duration of POD}

Nineteen $(45.2 \%)$ participants developed POD while hospitalized, with a median duration of 5 days (IQR 2-9). Delirium occurred mostly during the ICU stay $(\mathrm{n}=18,95 \%)$ and within the first 5 days post-LT. Two patients were discharged from the ICU to the ward with ongoing POD, which disappeared within 1 day of transition. Only one developed POD on the ward. As a quality indicator, we checked the performance of the newly implemented POD assessment. Nurses' full adherence to the delirium guidelines (ie, POD assessment of each included patient three times daily) would have resulted in 2164 POD assessments. In practice, POD was assessed 1643 times $(75.9 \%)$.

\section{Clinical Outcomes and Health-Care Utilization}

Ten $(23.8 \%)$ patients died within the follow-up time, whereof 6 $(60 \%)$ had experienced POD during hospital stay. Patients with POD had a shorter mean survival time than those who had not (585 days $[\mathrm{SD}=80.3]$ vs 742 days $[\mathrm{SD}=53.6]$ ), which was significant with the Breslow test $(P=.046)$. The Log-Rank test yielded no significant results $(P=.150$; Figure 2$)$. Results for clinical patient outcomes and health-care utilization are presented in Table 3.

\section{Factors Potentially Favoring the Development of POD}

We noted several statistically significant precipitating factors potentially favoring POD. However, due to our small sample size, we also paid attention to the clinically meaningful differences observed in the predisposing factors (Table 4). Compared to patients who experienced no delirium, patients with POD had a higher MELD score, a higher prevalence of pre-LT diabetes mellitus and depression, an increased need for renal replacement therapy before and after LT, longer duration of mechanical ventilation, longer surgery duration, more blood transfusions, and higher volumes of administered opioids and sedatives but less immunosuppressive medication (except cyclosporine).

\section{Discussion}

To our knowledge, this is the first prospective cohort study to examine POD as a primary outcome in LT recipients using validated assessment instruments. Our results are based on POD assessment performed by bedside nurses under normal working conditions on the ICU and the ward. Nearly half of the sample experienced POD during their hospital stay, mainly in the ICU. Postoperative delirium was associated with increased health-care utilization and decreased survival time. Our analyses also indicated potential factors favoring POD development.

\section{Incidence and Duration of POD}

This study group's high incidence of POD in LT recipients is consistent with Wang et al's finding of $47.4 \%$ via a validated assessment instrument. ${ }^{12}$ Studies not using validated tools found lower rates from $9.8 \%$ to $26.7 \% .^{7-11,13}$ Considering delirium's characteristic fluctuations, a systematic assessment yields greater sensitivity, and ICU nurses and physicians underestimate the presence of delirium without using diagnostic instruments. ${ }^{22}$ Consistent with previous reports, highest POD incidence occurred early after LT. ${ }^{7,12}$ Our study's POD duration is comparable to previous results with a mean of 
Table 2. Demographic and Clinical Patient Characteristics.

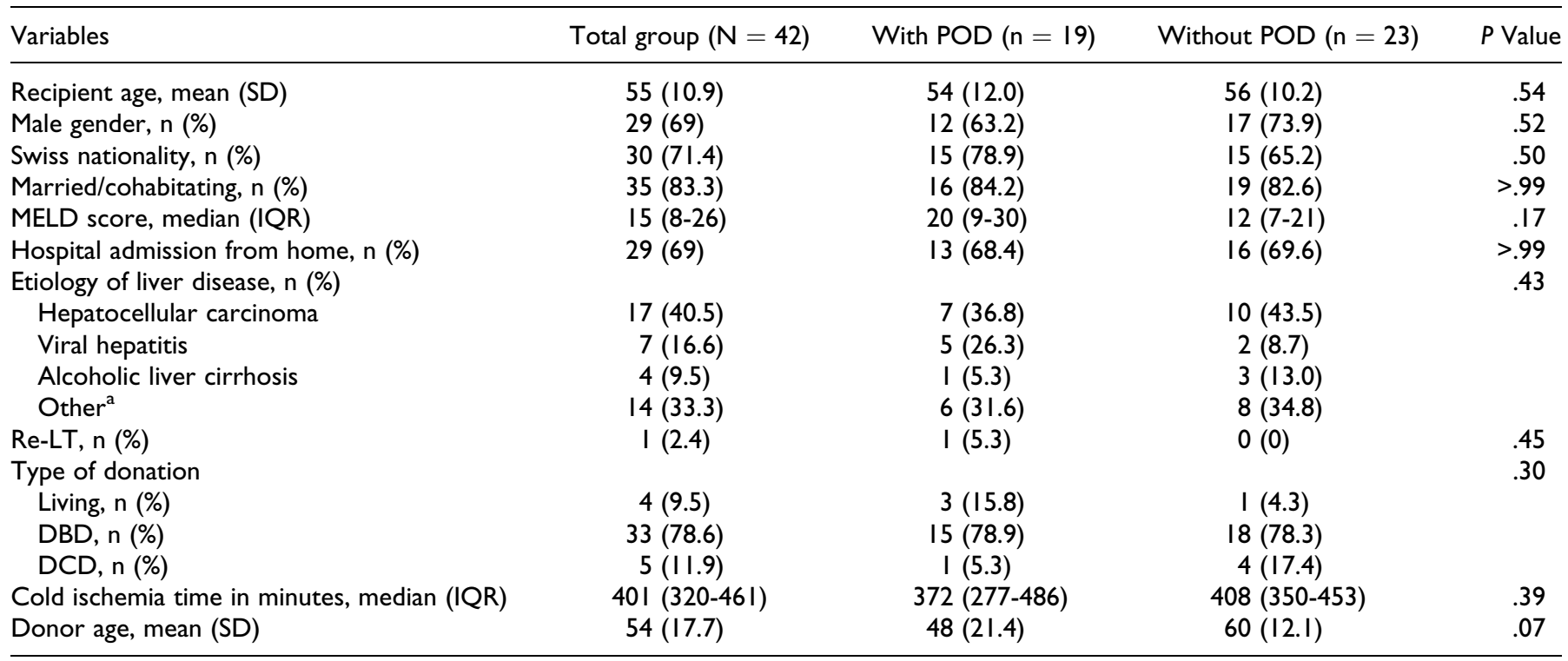

Abbreviations: POD, postoperative delirium; MELD, Model for End-Stage Liver Disease; LT, liver transplantation; SD, standard deviation; IQR, interquartile range; $\mathrm{DBD}$, donation after brain death; DCD, donation after cardiac death.

${ }^{\mathrm{a}}$ For example, Wilson disease, toxic cirrhosis, primary biliary cirrhosis, glycogenosis, and nonalcoholic steatohepatitis

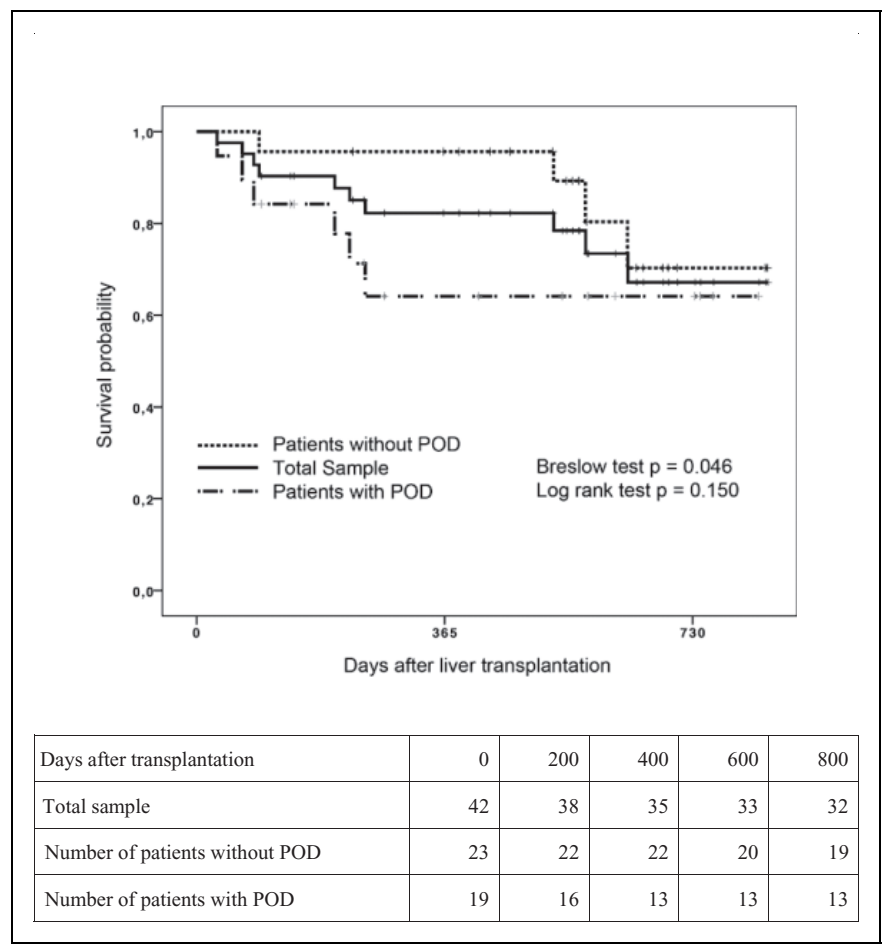

Figure 2. Kaplan-Meier survival curves of patients with and without POD. Patients were censored for not having experienced the event (death) at the end of study follow-up. The Wilcoxon-Breslow test is more sensitive to earlier events compared to the log-rank test. POD indicates postoperative delirium.

5 days $(\mathrm{SD}=2.6) .{ }^{12}$ Longer POD duration accompany serious risks: Alongside increased long-term functional decline and cognitive disorders, even a median POD duration of 1 to 3 days was associated with elevated 1-year mortality. ${ }^{23,24}$ In a sample of older ICU patients, Pisani et al concluded, "each day of delirium in the ICU increases the hazard of mortality by $10 \% . " 24(\mathrm{p} 1095)$

\section{Clinical Patient Outcomes}

Patients with POD had a decreased survival time compared to those without, which corroborates previous research. In a multivariate model adjusted for MELD scores, LT recipients with POD during ICU stay had a 4-fold higher risk of dying in hospital and a nearly 3 -fold higher 1 -year mortality. ${ }^{7}$ Our survival analysis showed that all 6 patients with POD during hospital stay died within the first year after LT, while 3 of 4 patients without POD died later than 1 year after LT. This also explains the significant difference in mean survival indicated by the Breslow test, which emphasizes earlier events. Concurring with previous findings, POD was also associated with increased health-care utilization. ${ }^{7,12}$ Prolonged hospitalization correlates with worse patient outcomes such as depression, reduced quality of life, and higher costs. ${ }^{25,26}$ Survival and health-care utilization are also influenced by other factors than POD though. For example, more than $50 \%$ of our recipients with POD also required post-LT renal replacement therapy, which has been associated with an increased length of stay and inferior patient and graft survival. ${ }^{27}$ Interestingly, although 11 (57.9\%) of the 19 patients with POD had severe complications or died during their hospital stay, we found no significant differences regarding postoperative complications.

\section{Factors Potentially Fostering POD Development}

We identified several clinical and statistical differences between patients with or without POD. We assume that 
Table 3. Comparison of Clinical Patient Outcomes and Health-Care Utilization Between Patients With and Without POD.

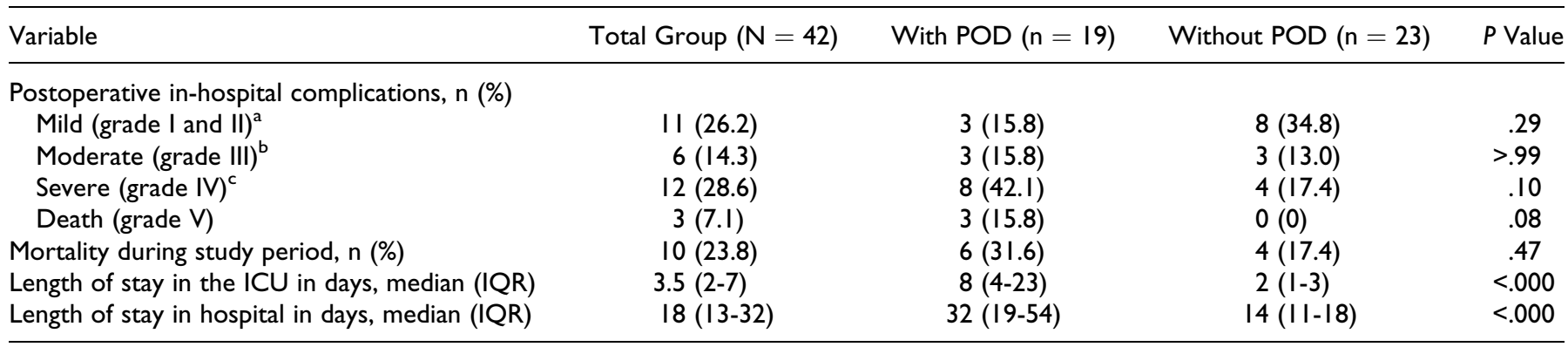

Abbreviations: POD, postoperative delirium; ICU, intensive care unit; IQR, interquartile range.

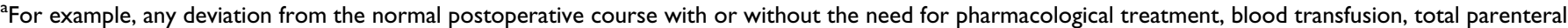
nutrition.

${ }^{b}$ For example, requiring surgical, endoscopic, or radiological intervention with or without general anesthesia

${ }^{c}$ For example, life-threatening complication, single or multiorgan dysfunction.

Table 4. Predisposing and Precipitating Risk Factors in Patients With and Without POD.

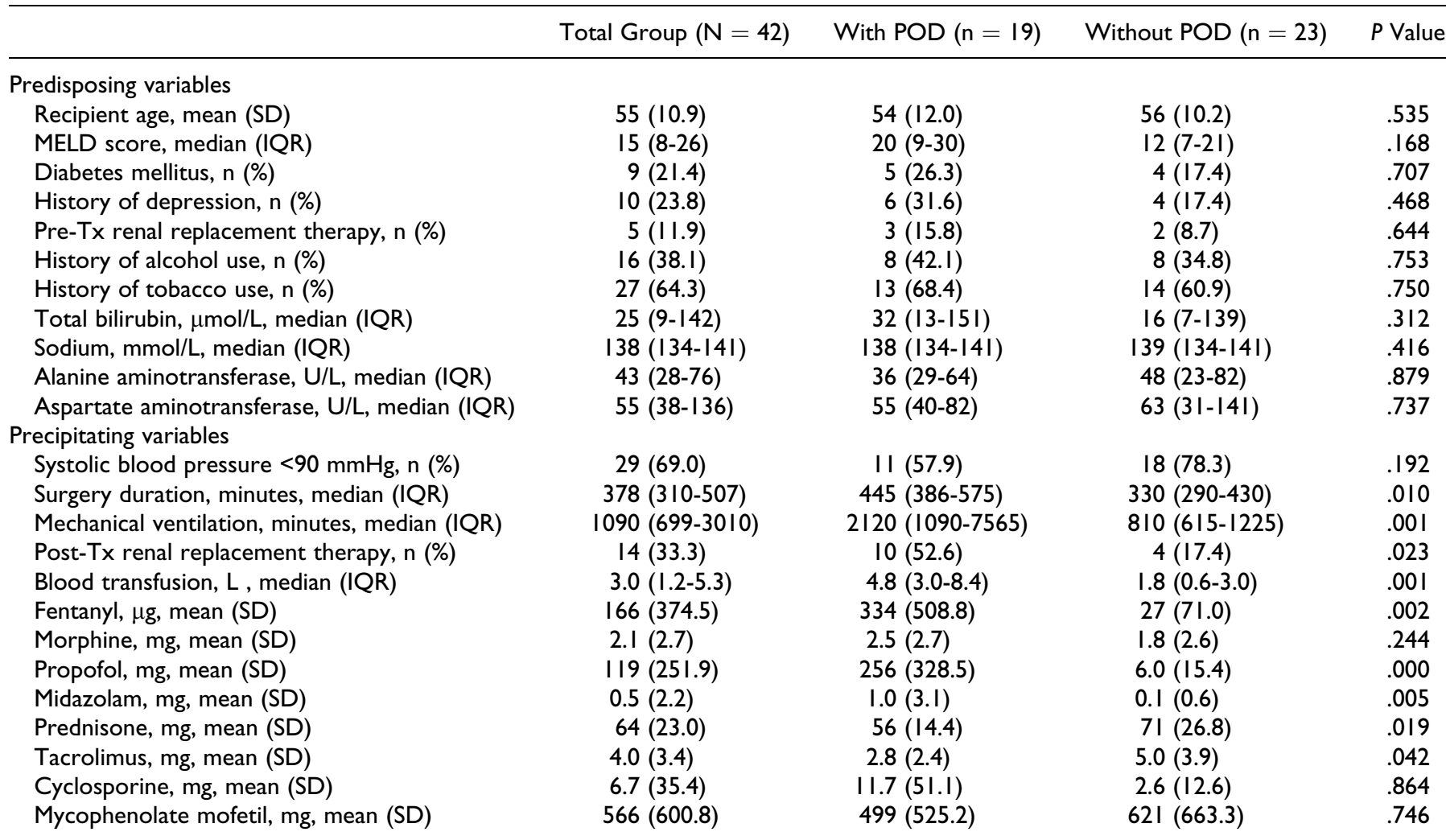

Abbreviations: POD, postoperative delirium; MELD, Model of End-Stage Liver Disease; LT, liver transplantation; SD, standard deviation; IQR, interquartile range.

predominantly pre-LT comorbidities and early intra- and postoperative factors might influence POD development. Comorbidities such as diabetes mellitus or depression have been associated with increased risk of POD after cardiac and hip fracture surgery. ${ }^{28,29}$ These comorbidities were also more prevalent in our patients with POD compared to those without. Our finding regarding the higher need for pre-LT renal replacement therapy in patients with POD is also supported by previous research. ${ }^{7}$ Notably, our patients with POD also showed a significantly increased need for post-LT renal replacement therapy, which has not yet been studied as a POD risk factor. Increased illness severity in our patients with POD was reflected in their higher MELD compared to those without POD. Although nonsignificant, this supports previous research. ${ }^{9,12}$ In contrast, Lescot et $\mathrm{al}^{7}$ reported the same MELD score in their patients with or without POD.

The clinical perspective suggests that some precipitating variables not only influence the development of POD per se but are also interrelated, as for example, longer surgery might require greater volumes of transfused blood. While 2 studies in 
LT found no significant results concerning surgery duration, ${ }^{7,12}$ we noted significant longer surgery in patients with POD, which may have entailed increased blood transfusion-a known POD risk factor. ${ }^{7}$ Additionally, an extended mechanical ventilation requires increased dosage of sedative medication. Being intubated $\geq 5$ days increased the risk of developing POD nearly 2 -fold, ${ }^{12}$ while benzodiazepines additionally contribute to the risk of delirium. ${ }^{30}$ Our patients with POD also had longer intubation time and received more sedative medication. However, sedatives are commonly administered during mechanical ventilation in the ICU and also prescribed to treat agitated, delirious patients. Therefore, firm conclusions concerning the relationship between medication and POD development should consider the time of administration, which fell beyond the scope of our research.

Other suspected precipitating factors were post-LT immunosuppressives. A common medication is tacrolimus. Although its neurotoxicity has been described, ${ }^{31}$ neurological complications in LT recipients might be influenced by multiple factors. ${ }^{11}$ However, tacrolimus is routinely discontinued in patients showing neurological symptoms or signs of POD. This most likely explains why our patients with POD received less tacrolimus than the non-POD group.

\section{Limitations}

Several limitations affected this study. First, systematic detection of POD was part of an interprofessional delirium management program implemented 1 month before data collection started. Although all nurses were appropriately trained to use the instruments, a lack of experience at the beginning may have led to POD rating errors. However, in our sensitivity analysis, we excluded patients with $<50 \%$ of the required 3 daily POD assessments and found no changes in patient characteristics or results regarding clinical outcomes, health-care utilization, or mortality. Second, POD diagnoses were not physician confirmed. Although we used validated instruments for POD assessment, the Intensive Care Delirium Screening Checklist's specificity is $57 \%$, allowing potential false-positive ratings. Additionally, since the Delirium Observation Screening Scale is a screening instrument, the diagnosis of POD requires confirmation with the Confusion Assessment Method, which represents key elements of delirium. ${ }^{32}$ Third, the small sample size limited the performance of multivariate statistics to determine independent predisposing and precipitating predictors of POD. Finally, although we carefully selected possible precipitating and predisposing factors for our population, further factors, for example, sleep deprivation, infections, or hepatic encephalopathy, may have contributed to POD in our sample.

\section{Clinical Implications}

The high incidence and long duration of POD indicated by our prospective and systematic assessment suggest that LT surgery entails a particularly high POD risk. Despite the necessary investments regarding training and administration, it is strongly recommended to incorporate systematic POD assessment with validated instruments into daily clinical care of LT recipients. Additionally, as POD frequently precedes severe negative clinical and economic outcomes, its relief and prevention must be set as high-priority goals. Multicomponent (non) pharmacological interventions can prevent or reduce the duration and severity of $\mathrm{POD}^{1}$ and should therefore be implemented in all centers performing LT.

\section{Conclusion}

In the current prospective study, more than $45 \%$ of LT recipients experienced POD, with a median duration of 5 days during their hospital stay. Postoperative delirium was associated with a 4-fold increase in ICU stay, a more than 2-fold increase in hospital stay, and decreased survival time. Postoperative delirium should not be considered as only predictive risk factor for worse outcomes in the LT population, but as POD is a preventable clinical complication, our results highlight the need for systematic assessment and the application of preventive (non) pharmacological interventions. The identification of risk factors for POD development would be an excellent subject for rigorous examination by future studies with larger sample sizes. Overall, given POD's strong associations with clinical outcomes in LT recipients, increasing our knowledge of it will speed the design and development of interventions targeted to shorten its duration, and, where possible, to prevent it entirely.

\section{Declaration of Conflicting Interests}

The author(s) declared no potential conflicts of interest with respect to the research, authorship, and/or publication of this article.

\section{Funding}

The author(s) received no financial support for the research, authorship, and/or publication of this article.

\section{References}

1. Inouye SK, Westendorp RG, Saczynski JS. Delirium in elderly people. Lancet. 2014;383(9920):911-922.

2. Dutkowski P, Oberkofler CE, Bechir M, et al. The model for endstage liver disease allocation system for liver transplantation saves lives, but increases morbidity and cost: a prospective outcome analysis. Liver Transpl. 2011;17(6):674-684.

3. Inouye SK, Charpentier PA. Precipitating factors for delirium in hospitalized elderly persons. Predictive model and interrelationship with baseline vulnerability. J Am Med Assoc. 1996;275(11): 852-857.

4. American Psychiatric Association. Diagnostic and Statistical Manual of Mental Disorders. 5th ed. Washington, DC: American Psychiatric Association; 2013.

5. Middle B, Miklancie M. Strategies to improve nurse knowledge of delirium: a call to the adult-gerontology clinical nurse specialist. Clin Nurse Spec. 2015;29(4):218-229.

6. De J, Wand AP. Delirium Screening: a systematic review of delirium screening tools in hospitalized patients. Gerontologist. 2015;55(6):1079-1099. 
7. Lescot T, Karvellas CJ, Chaudhury P, et al. Postoperative delirium in the intensive care unit predicts worse outcomes in liver transplant recipients. Can J Gastroenterol. 2013; 27(4):207-212.

8. Fukunishi I, Sugawara Y, Takayama T, Makuuchi M, Kawarasaki $\mathrm{H}$, Surman OS. Association between pretransplant psychological assessments and posttransplant psychiatric disorders in livingrelated transplantation. Psychosomatics. 2002;43(1):49-54.

9. Noma S, Hayashi A, Uehara M, et al. Psychosocial predictors of psychiatric disorders after living donor liver transplantation. Int J Psychiatry Clin Pract. 2008;12(2):120-126.

10. Chiu NM, Chen CL, Cheng ATA. Psychiatric consultation for post-liver-transplantation patients: regular article. Psychiatry Clin Neurosci. 2009;63(4):471-477.

11. Burkhalter EL, Starzl TE, Van Thiel DH. Severe neurological complications following orthotopic liver transplantation in patients receiving FK 506 and prednisone. J Hepatol. 1994; 21(4):572-577.

12. Wang SH, Wang JY, Lin PY, et al. Predisposing risk factors for delirium in living donor liver transplantation patients in intensive care units. PloS One. 2014;9(5):e96676.

13. Pinero F, Mendizabal M, Quiros R, et al. Neurological events after liver transplantation: a single-center experience. Transpl Int. 2014;27(12):1244-1252.

14. Swisstransplant. Schweizerische Nationale Stiftung für Organspende und Transplantation. Jahresbericht. 2014. https://www. swisstransplant.org/de/swisstransplant/publikationen/jahresbe richte/.

15. Bergeron N, Dubois MJ, Dumont M, Dial S, Skrobik Y. Intensive Care Delirium Screening Checklist: evaluation of a new screening tool. Intensive Care Med. 2001;27(5):859-864.

16. Radtke FM, Franck M, Oppermann S, et al. The Intensive Care Delirium Screening Checklist (ICDSC) - translation and validation of intensive care delirium checklist in accordance with guidelines. Anästhesiol Intensivmed Notfallmed Schmerzther. 2009; 44(2):80-86.

17. Sessler CN, Gosnell MS, Grap MJ, et al. The Richmond Agitation-Sedation Scale: validity and reliability in adult intensive care unit patients. Am J Respir Crit Care Med. 2002;166(10): 1338-1344.

18. Schuurmans MJ, Shortridge-Baggett LM, Duursma SA. The Delirium Observation Screening Scale: a screening instrument for delirium. Res Theory Nurs Pract. 2003;17(1):31-50.
19. Schuurmans MJ, Donders RT, Shortridge-Baggett LM, Duursma SA. Delirium case finding: pilot testing of a new screening scale for nurses. J Am Geriatr Soc. 2002;50(suppl A8):S3.

20. Dindo D, Demartines N, Clavien PA. Classification of surgical complications: a new proposal with evaluation in a cohort of 6336 patients and results of a survey. Ann Surg. 2004;240(2):205-213.

21. Breslow N. A generalized Kruskal-Wallis test for comparing $\mathrm{K}$ samples subject to unequal patterns of censorship. Biometrika. 1970;57(3):579-594.

22. Spronk PE, Riekerk B, Hofhuis J, Rommes JH. Occurrence of delirium is severely underestimated in the ICU during daily care. Intensive Care Med. 2009;35(7):1276-1280.

23. van den Boogaard M, Schoonhoven L, Evers AW, van der Hoeven JG, van Achterberg T, Pickkers P. Delirium in critically ill patients: impact on long-term health-related quality of life and cognitive functioning. Crit Care Med. 2012;40(1):112-118.

24. Pisani MA, Kong SY, Kasl SV, Murphy TE, Araujo KL, Van Ness PH. Days of delirium are associated with 1-year mortality in an older intensive care unit population. Am J Respir Crit Care Med. 2009;180(11):1092-1097.

25. Rattray JE, Hull AM. Emotional outcome after intensive care: literature review. J Adv Nurs. 2008;64(1):2-13.

26. Oeyen SG, Vandijck DM, Benoit DD, Annemans L, Decruyenaere JM. Quality of life after intensive care: a systematic review of the literature. Crit Care Med. 2010;38(12):2386-2400.

27. Rossi AP, Vella JP. Acute Kidney Disease After Liver and Heart Transplantation. Transplantation. 2016;100(3):506-514.

28. Lin Y, Chen J, Wang Z. Meta-analysis of factors which influence delirium following cardiac surgery. J Card Surg. 2012;27(4): 481-492.

29. Oh ES, Li M, Fafowora TM, et al. Preoperative risk factors for postoperative delirium following hip fracture repair: a systematic review. Int J Geriatr Psychiatry. 2015;30(9):900-910. doi:10. 1002/gps.4233.

30. Clegg A, Young JB. Which medications to avoid in people at risk of delirium: a systematic review. Age Ageing. 2011;40(1):23-29.

31. Benten D, Staufer K, Sterneck M. Orthotopic liver transplantation and what to do during follow-up: recommendations for the practitioner. Nat Clin Pract Gastroenterol Hepatol. 2009;6(1):23-36.

32. Inouye SK, van Dyck CH, Alessi CA, Balkin S, Siegal AP, Horwitz RI. Clarifying confusion: the confusion assessment method. A new method for detection of delirium. Ann Intern Med. 1990;113(12):941-948. 